\title{
Sustained absorption of delamanid from lipid-based formulations as a path to reduced frequency of administration
}

\author{
Gisela Ramirez $^{1} \cdot$ Anna C. Pham ${ }^{1} \cdot$ Andrew J. Clulow $^{1} \cdot$ Malinda Salim $^{1} \cdot$ Adrian Hawley $^{2} \cdot$ Ben J. Boyd $^{1,3}$ (D)
}

Accepted: 1 September 2020 / Published online: 15 September 2020

(C) The Author(s) 2020

\begin{abstract}
Delamanid is a poorly water-soluble drug currently being used for the treatment of tuberculosis. The high frequency of dosing leads to poor adherence for patients who live in lower economic and nomadic populations. Non-digestible self-assembling lipids as a formulation approach for poorly water-soluble drugs have previously been shown to extend the window of absorption through gastric retention. We hypothesise that this approach could lead to the reduction of dosing frequency for delamanid and thereby has potential to improve adherence. Formulations of delamanid were prepared in selachyl alcohol and phytantriol as nondigestible self-assembling lipid vehicles, and their behaviour was compared with reconstituted milk powder, as a digestible lipidbased formulation, and an aqueous suspension. The self-assembly of selachyl alcohol and phytantriol in aqueous media in the presence of delamanid was studied using small angle X-ray scattering and produced the inverse hexagonal $\left(\mathrm{H}_{2}\right)$ and inverse bicontinuous cubic $\left(\mathrm{V}_{2}\right)$ liquid crystal structures, respectively. The times at which maximum delamanid levels in plasma were observed $\left(\mathrm{T}_{\max }\right)$ after oral administration of the phytantriol, selachyl alcohol and reconstituted milk powder formulations of delamanid to rats were $27 \pm 3,20 \pm 4$ and $6.5 \pm 1.0 \mathrm{~h}$, respectively, compared with the aqueous suspension formulation with a $\mathrm{T}_{\max }$ of $3.4 \pm 1 \mathrm{~h}$, which confirms the hypothesis of an extended duration of absorption after administration in non-digestible selfassembling lipids. The digestion products of the triglycerides in the milk formulation increased the solubilisation of delamanid in the gastrointestinal tract, leading to an increase in exposure compared with the aqueous suspension formulation but did not significantly extend $\mathrm{T}_{\max }$. Overall, the non-digestible nanostructured lipid formulations extended the duration of absorption of delamanid well beyond that from milk or suspension formulations.
\end{abstract}

Keywords Delamanid - Sustained release - Lipid formulation - Lyotropic liquid crystal - Small angle X-ray scattering · Pharmacokinetics

Electronic supplementary material The online version of this article (https://doi.org/10.1007/s13346-020-00851-z) contains supplementary material, which is available to authorized users.

Ben J. Boyd

ben.boyd@monash.edu

1 Drug Delivery, Disposition and Dynamics, Monash Institute of Pharmaceutical Sciences, Monash University, Parkville Campus, 381 Royal Parade, Parkville, VIC 3052, Australia

2 SAXS/WAXS beamline, Australian Synchrotron, ANSTO, 800 Blackburn Rd, Clayton, VIC 3169, Australia

3 ARC Centre of Excellence in Convergent Bio-Nano Science and Technology, Monash Institute of Pharmaceutical Sciences, Monash University, Parkville Campus, 381 Royal Parade,

Parkville, VIC 3052, Australia

\section{Introduction}

Tuberculosis (TB) caused by Mycobacterium tuberculosis remains one of the leading causes of mortality worldwide with over $95 \%$ of deaths occurring in low- and middle-income countries [1]. Current first-line treatments for TB typically consist of 6-9 months of oral administration of multiple drugs taken daily or several times a week; and drugs for second-line treatment are administered when resistance to the first-line TB drugs has been diagnosed. Delamanid (previously known as OPC-67683, see Fig. 1 for chemical structure) is a nitrodihydro-imidazooxazole derivative known to exhibit potency against multidrug-resistant tuberculosis (MDR-TB) strains [2, 3], which can be used in a combination drug treatment for MDR-TB. The recommended dose of commercially available delamanid (Deltyba) in adults is $100 \mathrm{mg}$ daily (two tablets twice a day) for a 6-month period of treatment administered 


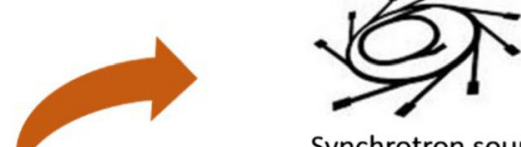

Synchrotron source small angle $\mathrm{X}$-ray scattering

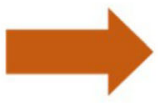

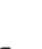

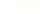

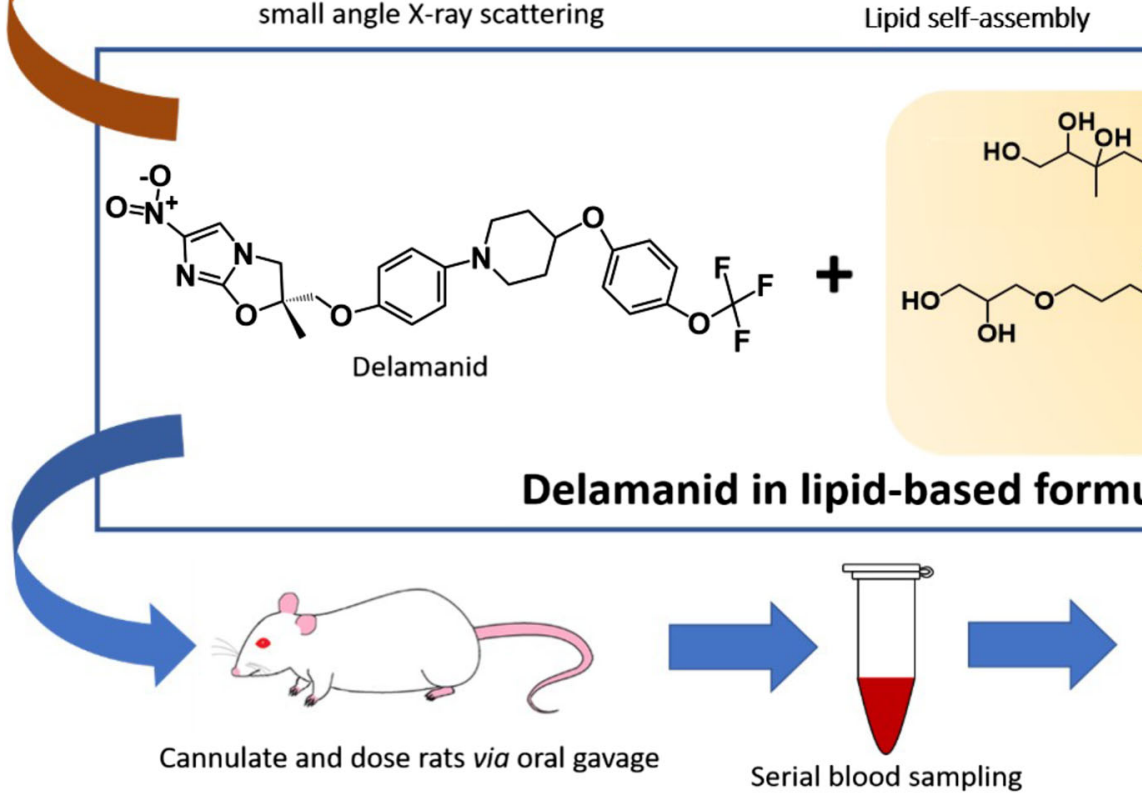

Phytantriol

\section{Correlation with oral bioavailability?}


purchased from DSM Nutritional Products (Singapore). Selachyl alcohol (1-O-octadec-9-enyl glycerol; SA; 99\% purity) was purchased from Haihang Industry Co., Ltd., (Jinan, China). All lipids were used without further purification. Devondale full cream instant milk powder (trademark of Murray Goulburn Co-operative Co. Ltd.) was purchased from a local supermarket (VIC, Australia). Tween 80, carboxymethylcellulose, Trizma ${ }^{\circledR}$ maleate (reagent grade), 4bromophenylboronic acid (4-BPBA, > 95\% purity), sodium taurodeoxycholate hydrate (NaTDC, $>95 \%$ purity) and diazepam were purchased from Sigma-Aldrich (St. Louis, USA). Acetonitrile (HPLC grade), methanol (LiChrosolv) and glacial acetic acid were purchased from Merck Millipore (Bayswater, Australia). Hydrochloric acid (36\%) was purchased from Biolab Ltd. (VIC, Australia). Calcium chloride dihydrate (>99\% purity) and sodium hydroxide pellets (min. 97\% purity) were purchased from Ajax Finechem (NSW, Australia). Sodium chloride ( $>99 \%$ purity) was purchased from Chem Supply (SA, Australia). USP grade pancreatin extract was purchased from Southern Biologicals (VIC, Australia). Sodium heparin $(1000 \mathrm{IU} / \mathrm{mL})$ was purchased from DBL (VIC, Australia). Saline $(0.9 \%$ sodium chloride solution) was purchased from Baxter Healthcare Pty. Ltd. (NSW, Australia). Lethabarb $(325 \mathrm{mg} / \mathrm{mL}$ solution of pentobarbitone sodium) was purchased from Abbott Laboratories Pty. Ltd. (NSW, Australia). DOPC (1,2-dioleoyl-sn-glycero3-phosphatidylcholine) was purchased from Sapphire Bioscience Pty. Ltd. (NSW, Australia). Distilled and deionised water $\left(18.2 \mathrm{M} \Omega / \mathrm{cm}\right.$ at $\left.25^{\circ} \mathrm{C}\right)$ was obtained from a Millipore water purification system (Billerica, USA).

\section{Self-assembly behaviour of phytantriol and selachyl alcohol: SAXS studies}

Bulk PHY and SA (about $30 \mathrm{mg}$ ) were added to $1 \mathrm{~mL}$ of water, $0.1 \mathrm{M} \mathrm{HCl}$ solution (simulated gastric condition) or bile salt micelle solution (simulated small intestinal condition) with and without delamanid (drug/lipid ratio of 1:10 w/w, selected as a compromise between analytical sensitivity and volume of administration in later in vivo studies), and the samples were incubated at $37^{\circ} \mathrm{C}$ for $48 \mathrm{~h}$. The bile salt micelle solution (4.70 mM NaTDC and $0.98 \mathrm{mM}$ DOPC), based on infant fasted conditions [13], was prepared in Tris buffer (50 $\mathrm{mM}$ trizma maleate, $5 \mathrm{mM}$ calcium chloride dihydrate, $150 \mathrm{mM}$ sodium chloride and $6 \mathrm{mM}$ sodium azide) at $\mathrm{pH}$ 6.5, described in significant detail previously [14]. The self-assembled structures of PHY and SA in the three aqueous solutions were characterised using the SAXS/WAXS beamline at the Australian Synchrotron (ANSTO, VIC, Australia) [15]. Samples were loaded onto a 96-well temperature-controlled plate and sealed using Kapton (polyimide) tape. The plate (held at $37^{\circ} \mathrm{C}$ ) was mounted in the path of the X-ray beam (photon energy: $12 \mathrm{keV}$ and wavelength:
$1.033 \AA$ ), and measurements were taken with a $1 \mathrm{~s}$ acquisition time. A Pilatus $1 \mathrm{M}(170 \mathrm{~mm} \times 170 \mathrm{~mm})$ detector, located approximately $1540 \mathrm{~mm}$ from the sample position, was used to generate two-dimensional SAXS patterns, which were reduced to scattering functions plotted as intensity (I) versus scattering vector $(q=(4 \pi / \lambda) \sin \theta$ where $\lambda=\mathrm{X}$-ray wavelength and $2 \theta=$ scattering angle) using Scatterbrain software version 2.71 developed in-house at the Australian Synchrotron. Suspensions of delamanid in water and the simulated gastric and intestinal solutions containing no PHY or SA were also analysed. Samples were loaded into $1.5 \mathrm{~mm}$ outer diameter glass capillaries (Charles Supper, Westborough, MA, USA) and placed on a temperature-controlled capillary holder maintained at $37^{\circ} \mathrm{C}$. SAXS measurements were performed using the parameters described above.

\section{Solubility of delamanid in lipid-based formulations}

The solubility of delamanid in PHY, SA and milk (9\% fat to match the dose of PHY/SA, prepared by reconstituting milk powder in Tris buffer) was measured to estimate drug loading for the in vivo pharmacokinetic studies. To determine the equilibrium solubility of delamanid in PHY and SA, excess delamanid was added into microcentrifuge tubes containing molten PHY and SA (prepared by incubating the lipids at $37^{\circ} \mathrm{C}$ ). The lipid + drug mixtures were vortexed and incubated at $37^{\circ} \mathrm{C}$ on a roller mixer. Samples were removed after 4, 7 and 10 days of incubation, and centrifuged at $16,162 \mathrm{~g}$ for $30 \mathrm{~min}$. Approximately $100 \mathrm{mg}$ of the upper lipid layer was transferred into ultracentrifuge tubes, and the samples were recentrifuged at $434,902 \mathrm{~g}$ for $60 \mathrm{~min}$ at $37^{\circ} \mathrm{C}$. About $20 \mathrm{mg}$ of the lipid supernatant layer was collected and dissolved in 1:1 tetrahydrofuran:acetonitrile volume ratio. The samples were further diluted with acetonitrile (1:1 volume ratio) prior to injection into a Shimadzu (Shimadzu, Kyoto, Japan) HPLC system consisting of a CBM-20A system controller, an LC$30 \mathrm{AD}$ solvent delivery module, a SIL-30AC auto-sampler and a CTO-20AC column oven coupled with an SPD-M30A diode array UV detector set to integrate at $254 \mathrm{~nm}$. Chromatographic separation of delamanid was performed at $35^{\circ} \mathrm{C}$ on a Waters Symmetry $\mathrm{C}_{18}$ column $(3.5 \mu \mathrm{m}, 100 \AA$, $4.6 \times 75 \mathrm{~mm}$ ). Delamanid was assayed using a binary gradient elution with a flow rate of $0.5 \mathrm{~mL} / \mathrm{min}$ : $30-90 \%$ B for $10 \mathrm{~min}$, 90-30\% B for 3 min and 30\% B for 4 min. Buffer A was $1 \%$ $\mathrm{v} / \mathrm{v}$ glacial acetic acid in water, and buffer B was $1 \% \mathrm{v} / \mathrm{v}$ glacial acetic acid in acetonitrile. Delamanid stock solutions (in $\mathrm{ACN}$ ) were used to provide a standard reference range of $0.1-40 \mu \mathrm{g} / \mathrm{mL}$ in $\mathrm{ACN}$. The injection volume was $10 \mu \mathrm{L}$ and the retention time for delamanid was $6.9 \mathrm{~min}$.

To determine the equilibrium solubility of delamanid in high fat $(9 \% w / v)$ reconstituted milk, excess delamanid was added to the milk. The drug + milk mixtures were vortexed and incubated at $4{ }^{\circ} \mathrm{C}$ with constant stirring. Samples $(200 \mu \mathrm{L})$ 
were removed at specified time points ( $3 \mathrm{~h}, 24 \mathrm{~h}$ and 3 days) and were placed into ultracentrifuge tubes and ultracentrifuged at $434,902 \mathrm{~g}$ for $60 \mathrm{~min}$ at $25^{\circ} \mathrm{C}$. The resultant aqueous, lipid and pellet layers were collected separately followed by extraction of delamanid using acetonitrile (for the aqueous layer) or 1:4 v/v methanol/acetonitrile (for the lipid and pellet layers) containing diazepam as the internal standard for HPLC. The amount of delamanid partitioned into the individual digested phases was quantified using the HPLC method described in the "Quantification of delamanid in plasma using HPLC” section.

\section{Bioavailability of delamanid in rats}

\section{Preparation of formulations for oral administration}

Delamanid was loaded into bulk PHY, bulk SA and milk, at a drug/lipid ratio of 1:10 w/w and a fixed dose of $10 \mathrm{mg}$ delamanid per kilogram of rat. Aqueous suspensions of delamanid (containing $0.5 \% \mathrm{v} / \mathrm{v}$ sodium carboxymethylcellulose and $0.4 \% \mathrm{v} / \mathrm{v}$ Tween 80 in saline) were also prepared as control formulations. The drug aqueous suspensions and the lipid-containing formulations were equilibrated for $24 \mathrm{~h}$ at $37^{\circ} \mathrm{C}$ prior to dosing.

\section{Animal procedures and sample collection}

All animal studies were approved and conducted in accordance with the guidelines of the Monash Institute of Pharmaceutical Sciences Animal Ethics Committee. Male Sprague Dawley rats (280-320 g) were used for the pharmacokinetic studies, and the rats were divided into 4 treatment groups with 4 rats per treatment: delamanid in aqueous suspension, PHY, SA and milk formulations. Rats were anaesthetised via inhalation of $5 \%$ isoflurane (and maintained at $2 \%$ for the duration of the procedure). The right carotid artery was isolated and cannulated with a $0.80 \mathrm{~mm} \mathrm{OD} \times$ $0.50 \mathrm{~mm}$ ID polyethylene tubing to allow serial blood sampling. Cannulas were filled and flushed with $10 \mathrm{IU} / \mathrm{mL}$ heparin in normal saline to maintain cannula patency. The cannula was tunnelled to back of the neck. The rats were then placed in a tether swivel system in individual wire bottom metabolic cages to recover overnight prior to dosing and blood sampling. The rats were then fasted for at least $12 \mathrm{~h}$ prior to dosing and $8 \mathrm{~h}$ after dosing, with water provided ad libitum. The lipid formulations and the saline suspensions were dosed via oral gavage $(225 \mu \mathrm{L})$, with the dose accurately calculated by weighing the gavage and syringe before and after dosing. Serial blood sampling was performed at 0.5, 1, 1.5, 2, 3, 4, $6,8,24,36,48,60,72$ and $96 \mathrm{~h}$ (up to $48 \mathrm{~h}$ for milk) with $250 \mu \mathrm{L}$ drawn at each time point. Blood samples were dispensed in $1.5 \mathrm{~mL}$ microcentrifuge tubes containing $10 \mathrm{IU}$ sodium heparin, which were then centrifuged for $5 \mathrm{~min}$ at
$6700 \mathrm{~g}$. Aliquots of plasma $(50 \mu \mathrm{L})$ were collected in duplicates, and samples were kept at $-20{ }^{\circ} \mathrm{C}$ until quantification of delamanid using HPLC.

\section{Quantification of delamanid in plasma using HPLC}

The collected plasma samples $(50 \mu \mathrm{L})$ were spiked with $10 \mu \mathrm{L}$ of diazepam solution $(10 \mu \mathrm{g} / \mathrm{mL}$ in acetonitrile) as an internal standard followed by the addition of $90 \mu \mathrm{L}$ acetonitrile. Samples were then centrifuged at $16,162 \mathrm{~g}$ for $7 \mathrm{~min}$ at room temperature, and $95 \mu \mathrm{L}$ of the supernatant were transferred to HPLC vials. Plasma standards were prepared by spiking delamanid stock solutions (in ACN) into plasma to give a standard reference range of 5-1000 ng/mL. The injection volume was $50 \mu \mathrm{L}$. Chromatographic separation of delamanid was performed using HPLC methods described in the "Solubility of delamanid in lipid-based formulations" section with slight modification to the elution gradient: 30 $50 \%$ B for $7 \mathrm{~min}, 50 \%$ B for $0.5 \mathrm{~min}, 50-70 \%$ B for $2.5 \mathrm{~min}$, $70 \% \mathrm{~B}$ for $1 \mathrm{~min}, 70-90 \% \mathrm{~B}$ for $1 \mathrm{~min}, 90-30 \%$ B for $1 \mathrm{~min}$ and $30 \% \mathrm{~B}$ for $4 \mathrm{~min}$. The retention time for diazepam was $9.6 \mathrm{~min}$ and for delamanid was $10.3 \mathrm{~min}$.

\section{Determination of pharmacokinetic parameters}

Concentrations of delamanid in plasma were dose-normalised to $10 \mathrm{mg} / \mathrm{kg}$ to account for variations in animal weight. Values of $\mathrm{C}_{\max }$ (maximum drug concentration in plasma) and $\mathrm{T}_{\max }$ (time to reach $\mathrm{C}_{\max }$ ) were determined from the normalised data. The truncated area under the curve (AUC) from 0 to the last measurable time points $\left(\mathrm{AUC}_{0 \text {-tlast }}\right)$ was determined using the trapezoidal rule. SPSS for Windows (Version 23) was used to statistically determine differences in the data using one-way ANOVA analysis of variance with Tukey's multiple comparison, assuming statistical significance when $p$ value was $\leq 0.05$.

\section{Solubilisation of delamanid in milk during in vitro lipolysis}

In vitro lipolysis of milk mixed with delamanid was performed to use the in vitro solubilisation data to interpret the bioavailability of delamanid. Delamanid $(22.5 \mathrm{mg})$ was added to $2.5 \mathrm{~mL}$ of water containing $0.25 \mathrm{~mL}$ of $1 \mathrm{M} \mathrm{HCl}$ solution. The drug sample was vortexed thoroughly prior to the addition of $17.5 \mathrm{~mL}$ of reconstituted powdered milk ( $9 \%$ fat in the Tris buffer) containing bile salt micelles with final concentrations of $4.7 \mathrm{mM}$ NaTDC and $0.98 \mathrm{mM}$ DOPC. The drug + milk mixture (22.5 mg delamanid/1.58 g milk fat) was incubated in a thermostated $\left(37^{\circ} \mathrm{C}\right)$ glass vessel under constant magnetic stirring. The $\mathrm{pH}$ of the mixture was adjusted to $6.500 \pm 0.005$ prior to the initiation of lipolysis with pancreatin lipase suspension $(2.25 \mathrm{~mL}, \sim 700 \mathrm{TBU} / \mathrm{mL}$ of digest $)$. The 
pancreatin lipase solution was prepared from pancreatin extract using methods previously described [16]. The $\mathrm{pH}$ of the sample in the digestion vessel was maintained at 6.5 during digestion using $2 \mathrm{M} \mathrm{NaOH}$ solution titrated into the digesta by a pH STAT control module (Metrohm AG, Herisau, Switzerland). Samples $(200 \mu \mathrm{L})$ were collected before ( $0 \mathrm{~min})$ and after digestion (60 $\mathrm{min})$ into ultracentrifuge tubes, and $2 \mu \mathrm{L}$ of lipase inhibitor ( $0.5 \mathrm{M} 4$-BPBA in methanol) was added into each sample before ultracentrifugation at $434,902 \mathrm{~g}$ for $1 \mathrm{~h}$ at $37^{\circ} \mathrm{C}$. The resultant aqueous, lipid and pellet layers were collected separately after ultracentrifugation and extraction of delamanid from the three separate layers were performed using methods described in the "Solubility of delamanid in lipid-based formulations" section. Delamanid concentrations in each separated layer were determined based on the standard curve made by spiking delamanid stock solutions (in ACN) in digested milk with concentrations ranging from $0.1-250 \mu \mathrm{g} / \mathrm{mL}$. The delamanid concentration was quantified using the HPLC method described in the "Quantification of delamanid in plasma using HPLC" section.

\section{Results and discussion}

\section{Self-assembly behaviour of phytantriol and selachyl alcohol in the presence of delamanid}

Formation of lipid liquid crystal structures in the gastrointestinal tract is known to dictate the partitioning of poorly watersoluble drugs and their oral bioavailability [9]. Characterising the self-assembly properties of PHY and SA in the gastrointestinal condition and the impact of incorporated drug is therefore important in understanding how these lipids behave during their passage through the gastrointestinal tract. The phase behaviour of bulk PHY and SA after exposure to different aqueous solution conditions (water, simulated gastric and simulated small intestinal conditions) was characterised using synchrotron SAXS to confirm the formation of lyotropic liquid crystalline structures due to self-assembly of the amphiphilic molecules. The scattering profiles in Fig. 2 show that an inverse bicontinuous cubic phase $\left(\mathrm{V}_{2}\right)$ with a $P n 3 m$ space group was formed after hydration of PHY in excess water as observed in previous studies [11, 19]. In comparison, SA selfassembled in water to form an inverse hexagonal phase [20] $\left(\mathrm{H}_{2}\right.$; relative peak ratios of $1, \sqrt{3}$ and $\left.\sqrt{4}\right)$ [17], although $\mathrm{H}_{2}$ phases with smaller lattice parameters relating to nonequilibrium structures were also present (see Table 1). These liquid crystal structures were not significantly impacted by the addition of delamanid. Similarly, no structural changes occurred following incubation of PHY and SA with and without delamanid at low $\mathrm{pH}$ (gastric) conditions, which was not unexpected considering the non-ionic nature of the lipids.

Figure 2 shows the influence of bile salt micelles (NaTDC/ DOPC) on the lyotropic liquid crystal structures formed by self-assembly of PHY and SA. Changes in structure of the $\mathrm{V}_{2}$ and $\mathrm{H}_{2}$ phases occurred following addition of surfactants at an intestinal $\mathrm{pH}$ of 6.5. For PHY, the $P n 3 m$ phase remained stable but there was an increase in lattice parameter of the $P n 3 m$ phase from 64 to $87 \AA$ (92 $\AA$ for PHY with delamanid). For SA, the $\mathrm{H}_{2}$ structure transformed into to a $P n 3 m$ cubic phase upon addition of bile salt micelles (Table 1). Addition of bile salts to PHY and SA therefore resulted in a less negative lipid curvature that could arise due to interaction between the bile acids and the lipid components, increasing the average size of the polar headgroups, and/or by decreasing the rigidity of the lipid bilayers [21]. It should be noted that the amount of bile salt micelles added to the aqueous buffer was within the concentration range generally reported for simulated human fasted intestinal fluid [22] and may not reflect the in vivo conditions in rats, in which higher concentrations of bile acids up to about $50 \mathrm{mM}$ have been observed [23]. The high concentrations of bile salts could therefore potentially further reduce the negative curvature of the lipids from $\mathrm{Pn} 3 m$ and $\mathrm{H}_{2}$ towards complete incorporation of PHY and SA into the bile salt mixed micelles, completely disrupting the inverse liquid crystalline phases.

\section{Pharmacokinetic studies of delamanid}

Mean concentrations of delamanid in plasma after oral administration in saline solution, PHY, SA and milk are shown in Fig. 3. Prolonged and increased levels of exposure to delamanid could be observed for all the lipid-based formulations compared with the saline suspension, particularly when administered with SA and PHY. The extent of drug exposure (determined by the area under the curve in the plasma concentration-time plot) and the maximum concentration of delamanid $\left(\mathrm{C}_{\max }\right)$ achievable at a specific time point $\left(\mathrm{T}_{\max }\right)$ are summarised in Table 2.

Overall, the relative bioavailability of the lipid formulations, calculated by the ratio of the AUC in the lipid formulations to delamanid aqueous saline suspension after dose normalisation, was highest for SA followed by PHY and milk. Analysis of the pharmacokinetic parameters using ANOVA revealed statistical differences in the $\mathrm{C}_{\max }, \mathrm{T}_{\max }$ and $\mathrm{AUC}_{0 \text {-last }}$ between PHY, SA, and delamanid suspensions with $p$ values $\leq 0.05$. However, no statistical differences were observed in the $\mathrm{T}_{\max }$ and $\mathrm{AUC}_{0 \text {-last }}(p$ values $>0.05$ ) between delamanid in the aqueous suspension and milk, although the mean $\mathrm{C}_{\max }$ for delamanid in milk was significantly greater.

As shown in Table 2, the mean $\mathrm{T}_{\max }$ was shortest when delamanid was administered in aqueous saline and milk suspensions. This indicated a rapid absorption of delamanid from 

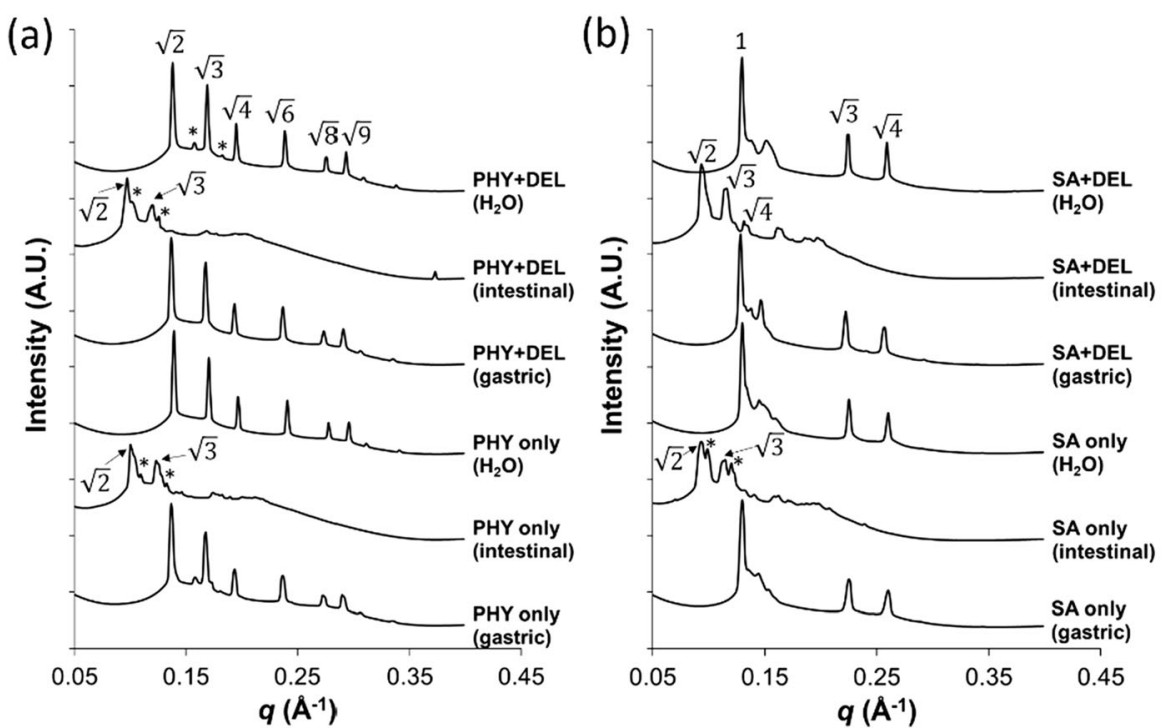

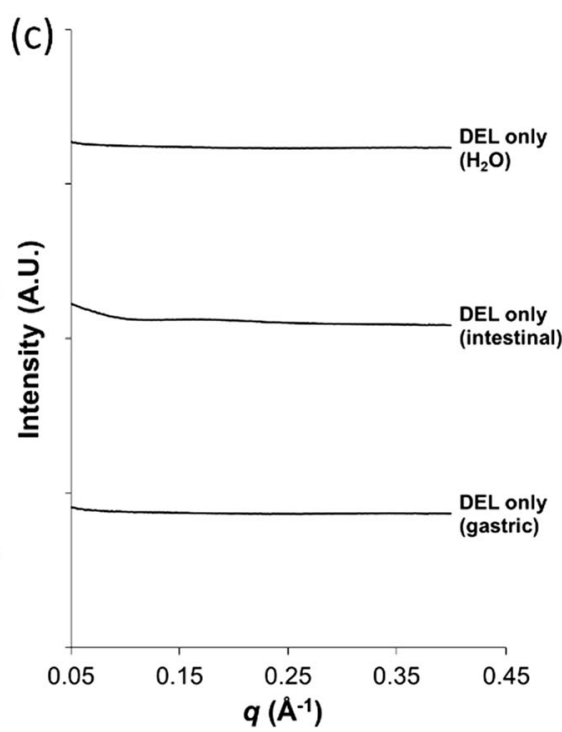

Fig. 2 Effect of aqueous environment and the presence of delamanid on the self-assembly of the lipid delivery vehicles measured using X-ray scattering. Panel a bulk phytantriol (PHY) and b selachyl alcohol (SA) in excess water, simulated gastric and small intestinal conditions in the absence and presence of delamanid (DEL). Panel $\mathbf{c}$ shows the X-ray scattering profiles for delamanid suspensions over the same range of scattering vector $q$. The ratio of the Bragg peaks for the $\mathrm{V}_{2}$ phase with $P n 3 m$ space group, and the $\mathrm{H}_{2}$ phase were identified. For $P n 3 m$, the peak ratios were $\sqrt{2}, \sqrt{3}, \sqrt{4}, \sqrt{6}, \sqrt{8}, \sqrt{9}$; and for $\mathrm{H}_{2}$ phase, the peak ratios were $1, \sqrt{3}$ and $\sqrt{4}[17,18]$. Peaks labelled with "*” were indicative of a $P n 3 m$ structures with different lattice parameters

to the formation of increasingly hydrophilic lipids during digestion. Digestion of the lipids in milk and the release of fatty acids, which have been previously shown to improve bioavailability of other poorly water-soluble drugs [24], were apparently insufficient to support the complete solubilisation of delamanid, despite the higher solubility of DEL in undigested milk compared with PHY and SA.

To further support the findings that digestion of milk did not result in improved drug solubilisation, in vitro digestion of delamanid in the reconstituted powdered milk (9\% fat w/v) was performed and the amount of drug partitioned into the
Table 1 Predominant liquid crystal structures and their corresponding lattice parameters for the self-assembly of phytantriol (PHY) and selachyl alcohol (SA) in excess water with and without delamanid at low $\mathrm{pH}$ (gastric) and intestinal $\mathrm{pH}$ containing bile salt micelles

\begin{tabular}{llll}
\hline System & Condition & Predominant structure & Lattice parameter $(\AA)$ \\
\hline PHY & Water & $P n 3 m$ & 64 \\
& Gastric & $P n 3 m$ & 65 \\
& Intestine & $P n 3 m$ & 87 \\
PHY + delamanid & Water & $P n 3 m$ & 64 \\
& Gastric & $P n 3 m$ & 65 \\
& Intestine & $P n 3 m$ & 92 \\
SA & Water & $\mathrm{H}_{2}$ & 55 \\
& Gastric & $\mathrm{H}_{2}$ & 56 \\
& Intestine & $P n 3 m$ & 95 \\
SA + delamanid & Water & $\mathrm{H}_{2}$ & 56 \\
& Gastric & $\mathrm{H}_{2}$ & 56 \\
& Intestine & $P n 3 m$ & 94 \\
\hline
\end{tabular}

Lattice parameters for the minor peaks in the SAXS profiles due to the presence of $\mathrm{V}_{2}$ phases with the Ia3d space group are described in Table $\mathrm{S} 1$ 
Table 2 Pharmacokinetic parameters (mean $\pm \operatorname{SEM}, n=4$ ) after oral administration of delamanid in aqueous suspension and delamanid in lipid formulations dosed at $10 \mathrm{mg}$ delamanid per kilogram of rat

\begin{tabular}{lllll}
\hline Formulation & $\begin{array}{l}\mathrm{T}_{\max } \\
(\mathrm{hr})\end{array}$ & $\begin{array}{l}\mathrm{C}_{\max } \\
(\mathrm{ng} / \mathrm{mL})\end{array}$ & $\begin{array}{l}\mathrm{AUC}_{0-\text { last }} \\
(\mathrm{hr} \mathrm{ng} / \mathrm{mL})\end{array}$ & Relative bioavailability (to suspension) \\
\hline Aqueous saline suspension & $3.4 \pm 1.0$ & $256 \pm 45$ & $5680 \pm 2530$ & 100 \\
Phytantriol & $27.0 \pm 3.0^{* * *}$ & $1055 \pm 29^{* * * *}$ & $31,600 \pm 2230^{* *}$ & 556 \\
Selachyl alcohol & $20.0 \pm 4.0^{* * *}$ & $1586 \pm 93^{* * * * *}$ & $36,600 \pm 5080^{* * *}$ & 645 \\
Milk & $6.5 \pm 1.0$ & $412 \pm 38$ & $9170 \pm 613$ & 162 \\
\hline
\end{tabular}

${ }^{*} p<0.05$ when compared with the saline suspension

$* * p<0.05$ when compared with the saline suspension and milk

$* * * p<0.05$ when compared with the saline suspension and selachyl alcohol

****p $<0.05$ when compared with the saline suspension, phytantriol and milk

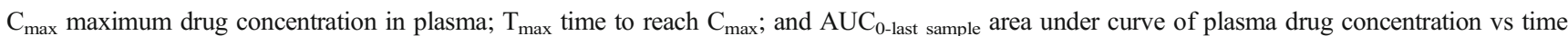
truncated from time 0 to the last time point sampled at $48 \mathrm{~h}$ for milk and $96 \mathrm{~h}$ for the other formulations

lipid and aqueous phases of the milk was quantified. Results in Fig. S1 showed that the amount of delamanid partitioned into the lipid and aqueous phases of milk before and after 60 min of digestion were not significantly different ( $p$ value $>0.05$ ). The solubilisation capacity of delamanid in the digested milk at the 60 min time-point was also not statistically significantly different to the equilibrium solubility of delamanid in $9 \%$ fat milk. It was therefore likely that digestion of milk did not improve the solubility of delamanid; hence, there was no substantial improvement in oral bioavailability using the milk formulation.

The pharmacokinetic profiles for PHY and SA showed that absorption of delamanid occurred for an extended period of time, and a significant increase in the oral bioavailability was realised. Delamanid was formulated in PHY and SA bulk lipid

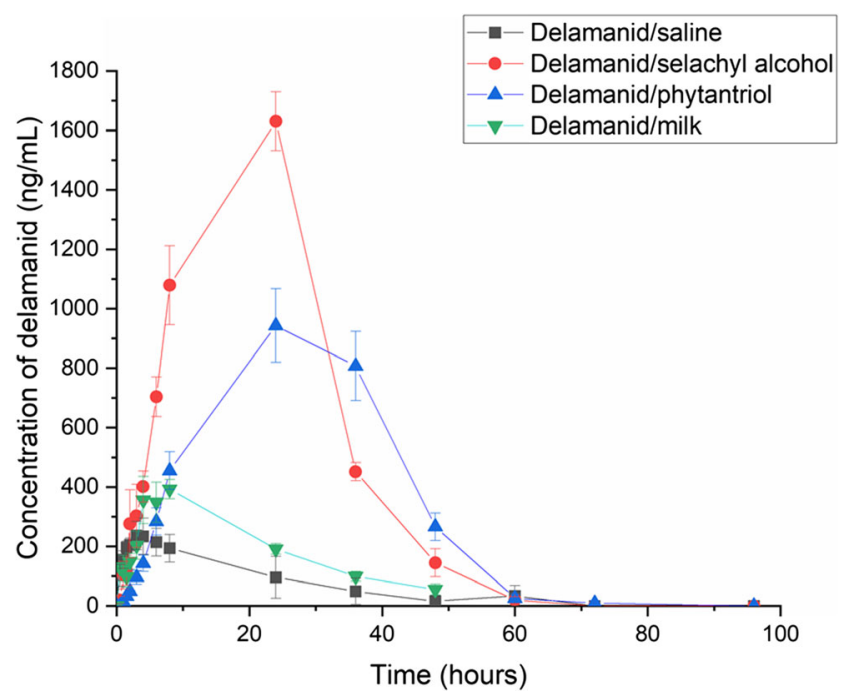

Fig. 3 Mean dose-normalised plasma concentrations of delamanid following oral administration in aqueous saline suspension and lipid-based formulations (phytantriol, selachyl alcohol and milk) over 96 h. Data are presented as mean $\pm \operatorname{SEM}(n=4)$ at a 10:1 lipid:drug mixture, which was in excess of the measured equilibrium solubilities. This meant that excess drug crystals were present due to low drug solubility. It has been previously demonstrated that PHY and SA are retained in the stomach for extended periods of time ( $>24 \mathrm{~h}$ for PHY) [10]. Previous studies with these lipids have typically had drug in solution in the lipid which resulted in $\mathrm{T}_{\max }$ values of $33.0 \pm$ 5.0 and $23.5 \pm 5.9 \mathrm{~h}$, respectively [10]. However, one study incorporated gold nanoparticles in PHY that were also retained in the stomach for extended periods ( $>8 \mathrm{~h}$ ) compared with a digestible lipid system when measured by X-ray computer tomographic imaging [25]. Thus it is not unexpected that a suspension of drug in these lipids is also likely to be retained for an extended period of time in the stomach. The chemical stability of PHY and SA against digestive enzymes appears to prevent these systems from exiting the stomach as quickly as digestible lipid systems or aqueous suspensions. The gastric compartment then has the potential to act in a non-sink condition, requiring drug released from the lipid suspension matrix to leave the stomach before further dissolution and partitioning can occur into the gastrointestinal fluids. Thus, the long duration of release can be understood through likely gastric retention already well demonstrated for these selfassembling lipid systems, but for the first time here with drug in suspension rather than fully dissolved.

Perhaps of greater interest are the differences in $\mathrm{C}_{\max }$ and AUC for the SA formulation compared with the PHY formulation. There is more drug in solution in the SA formulation than the PHY formulation by virtue of the greater solubility of delamanid in the host lipid, so it is likely that some lipid erosion occurs together with the drug release described above, leading to more drug arriving in the intestine in a dissolved state and commensurately greater absorption. The kinetic profiles are very similar, indicating that there are likely differences in the amount of drug in solution and available for absorption and that this drives the differences in plasma concentrations of drug over time. 
While the formation of self-assembled structures using non-digestible lipids has been previously shown to be critical in observing the long duration gastric retention behaviour, the type of liquid crystal structure formed does not appear to play a major role in the process. The self-assembly of the lipids under intestinal conditions was the same (both formed the $P n 3 m$ cubic phase under intestinal conditions, Table 1) so phase behaviour in the intestine is unlikely to have played a major role in the differences in overall exposure observed between the PHY and SA formulations. The similar shape of the kinetic profiles also suggests that the differences in phase behaviour in the gastric conditions (inverse hexagonal phase vs cubic phase) did not appear to play a significant role either. Thus, it is the propensity to form a liquid crystalline structure with non-digestible lipids, rather than the lipid and structure per se that appears to be important.

The PHY and SA formulations show a clear ability to provide enhanced drug exposure over at least a $24 \mathrm{~h}$ period. In the context of reducing pill burden and the potential for coformulation with a second once-daily drug in combination with delamanid, the lipid formulations studied here have potential to enable improved administration regimes including once daily dosing, which would provide an important opportunity to simplify treatment for tuberculosis in challenging populations. The two non-digestible lipids investigated here do not yet have GRAS status, although phytantriol [26] is a commercial cosmetic ingredient and selachyl alcohol [27] is an endogenous lipid also used in cosmetics. The oral LD50 values of phytantriol and related compounds are high > $2000 \mathrm{mg} / \mathrm{kg}$ in rats, so it is unlikely that they would show unusual toxicity behaviour; however, this remains to be determined in future formal preclinical and clinical safety studies.

\section{Conclusions}

This study showed the ability of phytantriol (PHY) and selachyl alcohol (SA) to sustain the release of delamanid from lipid formulations when administered in rats. The simple approach incorporates the non-digestible nature of lipids with prolonged gastric retention. In contrast, oral administration of delamanid with digestible lipids in the form of milk did not show sustained drug release characteristics. Comparable drug exposure between milk and an aqueous saline formulation was seen, which also highlighted the limited effect of milk fat digestion on the absorption of delamanid. Our findings suggested the potential use of non-digestible lipids as formulation strategies to improve the oral bioavailability of delamanid and extend the duration of drug exposure in plasma, with the potential to improve patient compliance by reducing the frequency of treatment.

Acknowledgements The authors thank Dr. Niya Bowers (Bill and Melinda Gates Foundation) for technical discussions and the Bill and
Melinda Gates Foundation for funding through OPP1160404. Andrew Clulow is supported through an Australian Research Council Discovery Early Career Research Award (DE190100531). SAXS measurements were conducted on the SAXS/WAXS beamline at the Australian Synchrotron, ANSTO.

Funding This study was supported by the Bill and Melinda Gates Foundation through OPP1160404.

\section{Compliance with ethical standards}

Conflict of interest The authors declare that they have no conflict of interest.

Open Access This article is licensed under a Creative Commons Attribution 4.0 International License, which permits use, sharing, adaptation, distribution and reproduction in any medium or format, as long as you give appropriate credit to the original author(s) and the source, provide a link to the Creative Commons licence, and indicate if changes were made. The images or other third party material in this article are included in the article's Creative Commons licence, unless indicated otherwise in a credit line to the material. If material is not included in the article's Creative Commons licence and your intended use is not permitted by statutory regulation or exceeds the permitted use, you will need to obtain permission directly from the copyright holder. To view a copy of this licence, visit http://creativecommons.org/licenses/by/4.0/.

\section{References}

1. World Health Organization: Tuberculosis. https://www.who.int/ news-room/fact-sheets/detail/tuberculosis. Accessed 7 Sept 2020.

2. Lewis JM, Sloan DJ. The role of delamanid in the treatment of drug-resistant tuberculosis. Ther Clin Risk Manag. 2015;11:77991.

3. Matsumoto M, Hashizume H, Tomishige T, Kawasaki M, Tsubouchi H, Sasaki H, et al. OPC-67683, a nitro-dihydroimidazooxazole derivative with promising action against tuberculosis in vitro and in mice. PLoS Med. 2006;3:e466.

4. Agency EM. Deltyba (Annex I: Summary of product characteristics). https://www.ema.europa.eu/en/medicines/human/EPAR/ deltyba\#product-information-section. Accessed 7 Sept 2020.

5. Wen H, Park K. Oral controlled release formulation design and drug delivery: theory to practice. Hoboken: Wiley; 2011.

6. Boyd BJ, Nguyen T-H, Müllertz A. Lipids in oral controlled release drug delivery. In: Controlled Release in Oral Drug Delivery. Wilson CG; Crowley PJ., Eds. Springer: NY; 2011. p. 299-327.

7. Pham AC, Peng K-Y, Salim M, Ramirez G, Hawley A, Clulow AJ, et al. Correlating digestion-driven self-assembly in milk and infant formulas with changes in lipid composition. ACS Appl Bio Mater. 2020;3:3087-98.

8. Clulow AJ, Salim M, Hawley A, Boyd BJ. A closer look at the behaviour of milk lipids during digestion. Chem Phys Lipids. 2018;211:107-16.

9. Boyd BJ, Khoo S-M, Whittaker DV, Davey G, Porter CJH. A lipidbased liquid crystalline matrix that provides sustained release and enhanced oral bioavailability for a model poorly water soluble drug in rats. Int J Pharm. 2007;340:52-60.

10. Nguyen T-H, Hanley T, Porter CJH, Boyd BJ. Nanostructured reverse hexagonal liquid crystals sustain plasma concentrations for a poorly water-soluble drug after oral administration. Drug Deliv Transl Res. 2011;1:429-38. 
11. Nguyen T-H, Hanley T, Porter CJH, Larson I, Boyd BJ. Phytantriol and glyceryl monooleate cubic liquid crystalline phases as sustained-release oral drug delivery systems for poorly watersoluble drugs II. In-vivo evaluation. J Pharm Pharmacol. 2010;62: $856-65$.

12. Larsson K. Two cubic phases in monoolein-water system. Nature. 1983;304:664.

13. Kamstrup D, Berthelsen R, Sassene P, Selen A, Müllertz A. In vitro model simulating gastro-intestinal digestion in the pediatric population (neonates and young infants). AAPS PharmSciTech. 2017;18:317-29.

14. Clulow AJ, Parrow A, Hawley A, Khan J, Pham AC, Larsson P, et al. Characterization of solubilizing nanoaggregates present in different versions of simulated intestinal fluid. J Phys Chem B. 2017;121:10869-81.

15. Kirby NM, Mudie ST, Hawley AM, Cookson DJ, Mertens HDT, Cowieson N, et al. A low-background-intensity focusing smallangle X-ray scattering undulator beamline. J Appl Crystallogr. 2013;46:1670-80.

16. Khan J, Rades T, Boyd BJ. Lipid-based formulations can enable the model poorly water-soluble weakly basic drug cinnarizine to precipitate in an amorphous-salt form during in vitro digestion. Mol Pharmaceut. 2016;13:3783-93.

17. Luzzati V, Husson F. The structure of the liquid-crystalline phases of lipid-water systems. J Cell Biol. 1962;12:207-19.

18. Tyler AI, Law RV, Seddon JM. X-ray diffraction of lipid model membranes. In: Methods in Membrane Lipids. Springer; 2015. p. 199-225.

19. Tilley A, Dong Y-D, Amenitsch H, Rappolt M, Boyd BJ. Transfer of lipid and phase reorganisation in self-assembled liquid crystal nanostructured particles based on phytantriol. Phys Chem Chem Phys. 2011;13:3026-32.
20. Younus M, Hawley A, Boyd BJ, Rizwan SB. Bulk and dispersed aqueous behaviour of an endogenous lipid, selachyl alcohol: Effect of Tween 80 and Pluronic F127 on nanostructure. Colloids Surf B: Biointerfaces. 2018;169:135-42.

21. Thurmond RL, Lindblom G, Brown MF. Effect of bile salts on monolayer curvature of a phosphatidylethanolamine/water model membrane system. Biophys J. 1991;60:728-32.

22. Riethorst D, Mols R, Duchateau G, Tack J, Brouwers J, Augustijns P. Characterization of human duodenal fluids in fasted and fed state conditions. J Pharm Sci. 2016;105:673-81.

23. Tanaka Y, Hara T, Waki R, Nagata S. Regional differences in the components of luminal water from rat gastrointestinal tract and comparison with other species. J Pharm Pharmaceut Sci. 2012;15: 510-8.

24. Boyd BJ, Salim M, Clulow AJ, Ramirez G, Pham AC, Hawley A. The impact of digestion is essential to the understanding of milk as a drug delivery system for poorly water soluble drugs. J Control Release. 2018;292:13-7.

25. Pham AC, Hong L, Montagnat O, Nowell CJ, Nguyen T-H, Boyd BJ. In vivo formation of cubic phase in situ after oral administration of cubic phase precursor formulation provides long duration gastric retention and absorption for poorly water-soluble drugs. Mol Pharmaceut. 2016;13:280-6.

26. Final report on the safety assessment of phytantriol. Int J Toxicol. 2007;26(Suppl 1):107-14.

27. Johnson W Jr, Bergfeld WF, Belsito DV, Hill RA, Klaassen CD, Liebler D, et al. Safety assessment of alkyl glyceryl ethers as used in cosmetics. Int J Toxicol. 2013;32(5 Suppl):5s-21s.

Publisher's note Springer Nature remains neutral with regard to jurisdictional claims in published maps and institutional affiliations. 